OPEN ACCESS

Edited by:

Wei Cao,

Shanghai Jiao Tong University, China

Reviewed by:

Zheqi Liu,

Shanghai Jiao Tong University, China

Yanjie Zhang,

Shanghai Jiao Tong University, China

${ }^{*}$ Correspondence.

Cheng Wang

wangch75@mail.sysu.edu.cn

Jiong Li

jli29@vcu.edu

${ }^{\dagger}$ These authors have contributed equally to this work

Specialty section:

This article was submitted to Head and Neck Cancer a section of the journal

Frontiers in Oncology

Received: 21 January 2021 Accepted: 25 March 2021 Published: 16 April 2021

Citation:

Wan Y, Hoyle RG, Xie N, Wang W, Cai $H$, Zhang $M$, Ma Z, Xiong G, Xu X,

Huang Z, Liu X, Li J and Wang C

(2021) A Super-Enhancer Driven by

FOSL 1 Controls miR-21-5p

Expression in Head and Neck

Squamous Cell Carcinoma.

Front. Oncol. 11:656628.

doi: 10.3389/fonc.2021.656628

\section{A Super-Enhancer Driven by FOSL1 Controls miR-21-5p Expression in Head and Neck Squamous Cell Carcinoma}

\author{
Yuehan Wan ${ }^{1,2+}$, Rosalie G. Hoyle ${ }^{3 \dagger}$, Nan Xie ${ }^{2,4}$, Wenjin Wang ${ }^{1,2}$, Hongshi Cai ${ }^{1,2}$, \\ Ming Zhang ${ }^{1,2}$, Zhikun Ma ${ }^{3}$, Gan Xiong ${ }^{1,2}$, Xiuyun $\mathrm{Xu}^{1,2}$, Zhengxian Huang ${ }^{1,2}$, \\ Xiqiang Liu ${ }^{5}$, Jiong $\mathrm{Li}^{3,6^{*}}$ and Cheng Wang ${ }^{1,2 *}$ \\ ${ }^{1}$ Department of Oral and Maxillofacial Surgery, Hospital of Stomatology, Guanghua School of Stomatology, Sun Yat-Sen \\ University, Guangzhou, China, ${ }^{2}$ Guangdong Provincial Key Laboratory of Stomatology, Sun Yat-Sen University, \\ Guangzhou, China, ${ }^{3}$ Department of Medicinal Chemistry, Institute for Structural Biology, Drug Discovery and Development, \\ School of Pharmacy and the Massey Cancer Center, Virginia Commonwealth University, Richmond, VA, United States, \\ ${ }^{4}$ Department of Oral Pathology, Guanghua School of Stomatology, Hospital of Stomatology, Sun Yat-Sen University, \\ Guangzhou, China, ${ }^{5}$ Department of Oral and Maxillofacial Surgery, Nanfang Hospital, Southern Medical University, \\ Guangzhou, China, ${ }^{6}$ Department of Oral and Craniofacial Molecular Biology, Philips Institute for Oral Health Research, \\ School of Dentistry, Virginia Commonwealth University, Richmond, VA, United States
}

MiR-21-5p is one of the most common oncogenic miRNAs that is upregulated in many solid cancers by inhibiting its target genes at the posttranscriptional level. However, the upstream regulatory mechanisms of miR-21-5p are still not well documented in cancers. Here, we identify a super-enhancer associated with the MIR21 gene (MIR21-SE) by analyzing the MIR21 genomic regulatory landscape in head and neck squamous cell carcinoma (HNSCC). We show that the MIR21-SE regulates miR-21-5p expression in different HNSCC cell lines and disruption of MIR21-SE inhibits miR-21-5p expression. We also identified that a key transcription factor, FOSL1 directly controls miR-21-5p expression by interacting with the MIR21-SE in HNSCC. Moreover, functional studies indicate that restoration of miR-21-5p partially abrogates FOSL1 depletion-mediated inhibition of cell proliferation and invasion. Clinical studies confirmed that miR-21-5p expression is positively correlated with FOSL1 expression. These findings suggest that FOSL1-SE drives miR-21-5p expression to promote malignant progression of HNSCC

Keywords: super enhancer, FOSL1, miR-21-5p, head and neck squamous cell carcinoma, metastasis

\section{INTRODUCTION}

Head and neck squamous cell carcinoma (HNSCC) is one of the most common malignant tumors worldwide, with a 5 -year survival rate of only about $50 \%(1,2)$. HNSCC easily invades surrounding tissues and spreads to cervical lymph nodes (LN), ultimately leading to relapse and death (3). Although a significant progress on the pathogenesis of HNSCC has been made in the past decades, the exact molecular mechanisms are still not well understood. Increasing evidences implicates that dysregulation of miRNAs has a critical role in the development and progression of HNSCC (4-10). 
In our previous studies, we also confirmed that miRNAs are involved in the tumor growth, stemness and metastasis of HNSCC by targeting a cohort of key oncogenes or tumor suppressors, including miR-21, miR-204-5p, miR320a, miR138 (11-13). Interestingly, the alterations of miRNA landscape showed that most of deregulated miRNA are downregulated in HNSCC as compared to normal tissue (14-17). Contrarily, only several upregulated miRNAs were identified, including miR-215p, miR-7 and miR-31 (18-22). Notably, miR-21-5p is one of the most common oncogenic miRNAs that is robustly upregulated and function as an oncogene in many solid cancers by inhibiting its target genes at the posttranscriptional level, including HNSCC $(23,24)$. However, the upstream regulatory mechanisms of miR21 are still not well documented in cancers.

Herein, we aimed to investigate the upstream regulatory mechanisms of miR-21-5p in HNSCC. Strikingly, a MIR21associated super enhancer (MIR21-SE) was identified in HNSCC and disruption of this super enhancer inhibits miR21-5p expression. Interestingly, a key transcription factor, FOSL1, was confirmed to directly promote miR-21-5p expression by interacting with the MIR21-SE in HNSCC. Taken together, these findings showed that the miR-21-5p expression was controlled by a FOSL1-driving-SE in HNSCC.

\section{MATERIALS AND METHODS}

\section{Samples}

95 OSCC tissues samples, 16 matched adjacent non-cancerous normal tissues (ANCT) were collected at the Department of Oral and Maxillofacial Surgery, Hospital of Stomatology, Sun Yat-sen University between January 2013 and July 2018. Tissue samples applied in this study were conducted in accordance with the Declaration of Helsinki's guidelines. All patients did not receive any form of preoperative treatment. The study was approved by the Ethical Committee of the hospital. Tumor grade was determined according to the 8th American Joint Committee on Cancer Staging System. Immunohistochemical and in situ hybridization analysis were used to assess the gene expression.

\section{Immunohistochemistry and In Situ Hybridization}

Formalin-fixed, paraffin-embedded tissue samples were cut into $4-\mu \mathrm{m}$ sections for immunohistochemistry analysis. The tissue sections were incubated at $4^{\circ} \mathrm{C}$ with primary antibodies against FRA1/FOSL1(1:100, ab232745, Abcam) overnight. Diamino benzidine (DAB, GK600510, Gene Tech, China) was used to visualize and hematoxylin (D006, Nanjing Jiancheng Biotech, China) was applied to counterstain. Two pathologists who were blinded to the clinical data evaluated the immunohistochemical stain independently. The intensity of FOSL1 staining was evaluated as 0 : no staining; 1 : weak; 2 : moderate; and 3 : strong. The proportion was evaluated as $0-100 \%$ positive cells. Staining index $($ SI: $0-300)=$ staining intensity $\times$ the proportion of positive cells. Samples with SI $>100$ were defined as "high expression" and those with SI $\leq 100$ were defined as "low expression".
miR-21-5p expression was examined by in situ hybridization according to the manufacturer's instructions (microRNA ISH Optimization Kit for FFPE, Exiqon, Vedbaek, Denmark). miR21-5p was hybridized with Double-DIG-labeled LNA ${ }^{\mathrm{TM}}$ microRNA probes (1:1250, Exiqon) overnight after demasking. The sections were blocked and incubated with goat antidigoxigenin-AP (1:1000, Roche) and then counterstained with nitro blue tetrazolium/5-bromo-4-chloro-3-indolylphosphate (NBT/BCIP). The staining intensity of the cells was recorded as follows: The intensity staining was evaluated as 0: no staining; 1: weak, light blue; 2: moderate, blue; and 3: strong, dark blue. The proportion was evaluated as 0 : negative; $1:<10 \% ; 2: 11-50 \%$; 3: $51-80 \%$ or $4:>80 \%$ positive cells. The staining index (SI) $=$ staining intensity $\times$ the proportion of positive cells. SI (miR-21$5 \mathrm{p})>4$ was defined as "high expression" and SI $\leq 4$ was defined as "low expression".

\section{Chromatin Immunoprecipitation Assay (ChIP)}

For ChIP assay, $10^{6}$ cells were treated with $1 \%$ formaldehyde at $37^{\circ} \mathrm{C}$ for 10 min to crosslink their DNA. Agarose gel electrophoresis was used to assess the DNA fragments between $500 \mathrm{bp}$ and $800 \mathrm{bp}$ the cells were lysed. The chromatin crosslink was incubated at $4^{\circ} \mathrm{C}$ overnight. Then purify the immunoprecipitated DNA after reversing the DNA-protein crosslink. Quantitative real-time PCR (qPCR) was used to quantify the final precipitated DNA. The PCR result was expressed with the percentage of input DNA. The primer sequences of ChIP-qPCR were listed as below. MIR21 super enhancer (MIR21-SE) 5'-AAACCACACTCTGTCG TATCTGTG-3' and 5'-TACAGAACGGCAAGAAAACTGGG-3' and negative control primer (MIR21-NEG) 5'- CCACCAT GCCCAGCCTTGAAGTTA-3' and 5'-TGGAGAGGGCT GACCTTAACCAA-3'.

\section{Luciferase Reporter Assay}

MIR21-SE and MIR21-NEG fragments were inserted into pLG4.23 luciferase reporter through Kpn1 and Xhol restriction sites using standard PCR-cloning method. The primer sequences for cloning MIR21-SE were 5'- GGGTACCGTTCTAGAAAAGAAGTGAA GGCCAGTCG-3' and 5' - CCTCGAGTTAGACATGCTT GCAGGCGTTT-3'. The primer sequences for cloning MIR21NEG were 5'- GGGTACCACCATGCCCAGCCTTGAAGTTAA-3’ and 5'- CCTCGAGACTGAGTGGGGAGAATTGCCTA -3'. For luciferase assay, SCC1 and 293T cells were plated in 24-well plates at $40-50 \%$ confluence. For luciferase assay in SCC1 cells, $12 \mathrm{~h}$ after seeding, the cells were transfected with FOSL1 siRNA or control siRNA. Then, after $24 \mathrm{~h}$, the cells were transfected with $50 \mathrm{ng}$ of luciferase reporters and $20 \mathrm{ng}$ of CMV-galactosidase constructs. For luciferase assay in $293 \mathrm{~T}$ cells, $12 \mathrm{~h}$ after seeding, the cells were transfected with $50 \mathrm{ng}$ of luciferase reporters, $20 \mathrm{ng}$ of CMVgalactosidase, and expression constructs of FOSL1 and JUN. The reporter activities were determined $24 \mathrm{~h}$ after DNA transfection. The luciferase and $\beta$-galactosidase activity of total cell lysates were determined by Bright-Glo Luciferase Assay System (Promega; cat\#E2620) and GalactoStar Reporter Gene Assay System (Applied Biosystems, cat\#T1012), respectively. The luciferase 
reporter activity was normalized against the $\beta$-galactosidase activity of each cell lysate sample.

\section{RNA Isolation and Quantitative Real-Time PCR (RT-qPCR)}

RNAzol $^{\circledR}$ RT (RN190, Molecular Research Center, USA) was used to extracted RNA from the cells per the manufacturer's protocol. $1 \mu \mathrm{g}$ of total RNA was applied to reverse transcription with the ribo SCRIPT Reverse Transcription kit (C11027, RIBOBIO, China). All miR-21-5p (RT primter: ssD809230239; qPCR primer: ssD809230931 and ssD089261711) and U6 (RT primter: ssD0904071008; qPCR primer: ssD0904071006 and ssD0904071007) RT-qPCR primers were ordered from RIBOBIO. The 3-step RT-qPCR reactions were carried out with a SYBR Green Master Mix (11201ES08, Yeasen, China) in the LightCycler 96 System (Roche, Germany). The thermal cycling parameters were as follows: preincubation at $95^{\circ} \mathrm{C}$ for $5 \mathrm{~min}$, followed by 40 cycles of amplification as: $95^{\circ} \mathrm{C}$ for $10 \mathrm{~s}$, $60^{\circ} \mathrm{C}$ for $20 \mathrm{~s}$, and $72^{\circ} \mathrm{C}$ for $20 \mathrm{~s}$, with a final cycle of $95^{\circ} \mathrm{C}$ for $15 \mathrm{~s}$, $60^{\circ} \mathrm{C}$ for $60 \mathrm{~s}$, and $95^{\circ} \mathrm{C}$ for $15 \mathrm{~s}$ as the melting curve. The relative expression level of miR-21-5p was calculated by $2-\Delta \Delta \mathrm{Ct}$. Human U6 was served as an internal reference.

\section{Cell Lines, Cell Culture, and Treatments}

SCC1 cells were obtained from University of Michigan and HN6 cells were obtained from Wayne State University. UM1 and UM2 cells were provided by Dr. Xiaofeng Zhou (University of Illinois at Chicago, IL, USA). SCC9, SCC15, SCC25 and CAL27 were obtained from the American Type Culture Collection (ATCC; Manassas, VA, USA). The SCC1, HN6 and CAL27 cell lines were cultivated in Dulbecco's modified Eagle's medium (DMEM, Gibco, USA) supplemented with $10 \%$ fetal bovine serum (FBS, Gibco, USA). The SCC9, SCC15, SCC25, UM1 and UM2 cell lines were grown in DMEM/F12 medium (DMEM/F12, Gibco, USA) supplemented with $10 \%$ FBS. Cells were kept at $37^{\circ} \mathrm{C}$ under a humidified atmosphere with 5\% CO2. Lipofectamine (Lipofectamine RNAiMAX Transfection Reagent, Thermo Fisher) was used to transfect miR-21-5p mimics (50nM, RIBOBIO), FOSL1 siRNA (100nM, RIBOBIO) and their respective negative controls. For the BET bromodomain Inhibitor treatment groups, cells were treated with $1 \mu \mathrm{mol} / \mathrm{mL}(+)-J Q 1$ (HY-13030, MCE, USA) or iBET151 (HY-13235, MCE, USA).

\section{Cell Proliferation}

HN6 and SCC1 cells were seeded in 96-well plates at a density of 1000 or 1500 cells per well. The cell confluence of untreated cells and treated cells were detected and analyzed using the Incucyte ${ }^{\circledR}$ S3 Live-Cell Analysis System (Essen BioScience, USA) at the indicated time points.

\section{Wound-Healing, Migration, and Invasion Assays}

Wound-healing assay was carried out with a sterile pipette tip to make scratches when cells reached $90 \%$ fusion degree in 6-well plates. FBS-free media was then used to cultivate cells for 48 h. $2 \times 10^{5}$ HN6 cells or $4 \times 10^{5}$ SCC 1 cells with $200 \mu$ l FBSfree media were seeded into the upper chambers (pore size: $8-\mu \mathrm{m}$, Corning, China) with or without Matrigel (354234, Corning, China) to verify the invasion or migration assays. $600 \mu \mathrm{l}$ of $10 \%$ fetal bovine serum (FBS) was added into the lower chambers. Cells stranded in the upper chambers after $24 \mathrm{~h}$ were removed, and the fixed cells on the lower surface of the membrane were stained with hematoxylin. Five random views were selected and photographed under a microscope (ZEISS, German). Image J was used to calculate the cell numbers and wound healing area.

\section{Statistical Analysis}

GraphPad prism 8.0. (GraphPad Software Inc.) was used for statistical analysis with the mean \pm standard deviation (SD). All statistical tests were two-tailed. P-values of $<0.05$ were considered statistically significant.

\section{RESULTS}

\section{miR-21-5p Is Upregulated and Correlated With Malignant Progression of HNSCC}

To investigate the potential role of miR-21-5p in HNSCC, we firstly evaluated miR-21-5p expression based on The Cancer Genome Atlas (TCGA) HNSCC datasets. As shown in Figure 1A, the expression of miR-21-5p was significantly increased in HNSCC as compared to normal tissue. To further validate these findings, we performed in situ hybridization to detect the expression of miR-21-5p in 95 HNSCC tissues. As expected, we found that miR-21-5p expression was mainly located in cytoplasm and significantly increased in HNSCC when compared to normal epithelium (Figures 1B, C). Moreover, the expression of miR-21-5p was also upregulated in HNSCC patient with T3,4 stage as compared to patients with T1,2 stage. Similar results were observed in HNSCC patient with lymph node metastasis as compared to patient without lymph node metastasis (Figures 1D, E). An increase of miR-21-5p was also observed in an 8 HNSCC cell lines panel as compared to NOK cells (Figure 1F).

To further investigate the functional role miR-21-5p in HNSCC, HNSCC cells, HN6 and SCC1 were transfected with miR-21-5p mimics and we found that cell proliferation and invasion were increased in cells treated with miR-21-5p mimics (Figures 1G-J). Taken together, these findings confirmed that miR-21-5p is upregulated in HNSCC and promotes malignant phenotypes in HNSCC.

\section{Targeting FOSL1 Suppresses miR-21-5p Expression by Interacting With the MIR21-SE}

To dissect the upstream regulatory machinery of miR-21-5p, we tried to analyze its promoter and enhancer region of the MIR21 

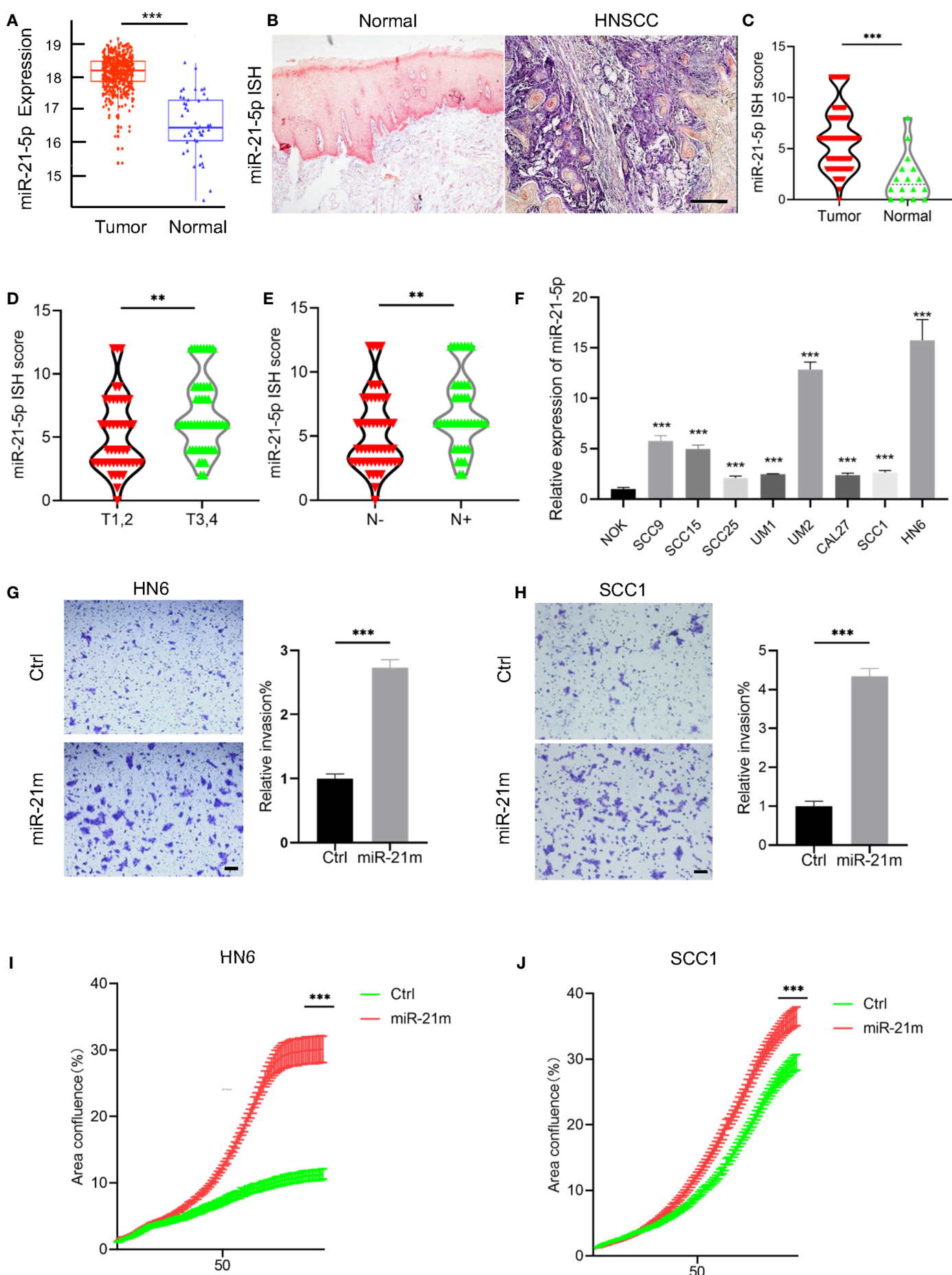

$\mathbf{J}$

SCC1

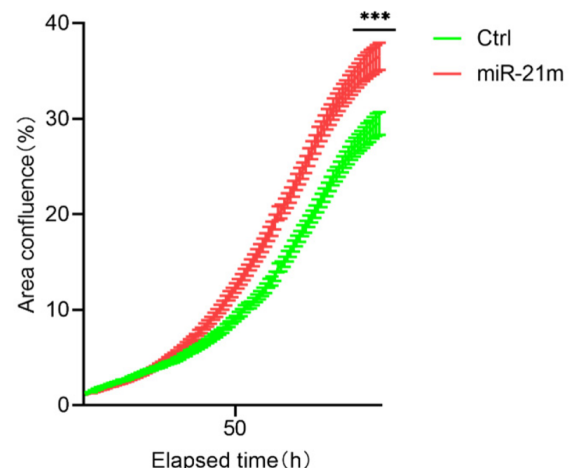

FIGURE 1 | The expression of miR-21-5p was upregulated and correlated with malignant progression in HNSCC. (A) The expression of miR-21-5p was upregulated in HNSCC as compared to the normal tissue. Data was from TCGA HNSCC datasets downloaded using the UCSC Xena browser (https://xenabrowser. net). ${ }^{* \star *} \mathrm{P}<0.001$. (B) The representative images of miR-21-5p ISH staining. Scale bar, 300 $\mu \mathrm{m}$ (C) The quantitative analysis of miR-21-5p ISH staining in HNSCC patient samples. ${ }^{* * *} \mathrm{P}<0.001$ by Student's t-test (D) The expression of miR-21-5p was increased in T3,4 stage HNSCC patient as comparted to those with T1,2 stage. ${ }^{* *} \mathrm{P}<0.01$ by Student's t-test (E) The expression of miR-21-5p was increased in HNSCC patient with lymph node metastasis as comparted to those without lymph node metastasis. ${ }^{* *} \mathrm{P}<0.01$ by Student's t-test $\mathbf{( F )}$ The relative of miR-21-5p was upregulated in 8 HNSCC cell lines as compared to NOK cells. ${ }^{* \star *} \mathrm{P}<0.001$ by Student t-test. (G, H) Overexpression of miR-21-5p promotes cell invasion of SCC1 and HN6 cells. ${ }^{* * *} \mathrm{P}<0.001$ by Student's t-test. Scale bar, $200 \mu \mathrm{m}$ (I, J) Overexpression of miR-21-5p promotes proliferation of SCC1 and HN6 cells. ${ }^{* \star} \mathrm{P}<0.001$ by two-way ANOVA 
gene based on our MED1 ChIP-seq results of human SCC cells from a different study (Figure 2A). Unexpectedly, we discovered that SEs were associated with MIR21. In agreement with ChIPseq findings, RT-qPCR showed that the expression of miR-21-5p was decreased in SCC1 and HN6 cells upon JQ1 and iBET-151 treatment (Figures 2B, C), two well-known BET inhibitors which can disrupt SE. Consistently, ChIP-PCR showed that the enrichments of MED1 and BRD4 on miR-21-SE region were also significantly suppressed in cells treated with JQ1 and iBET-151 (Figures 2D, E). Interestingly, the enrichment of FOSL1 in promoter region of MIR21 was also decreased upon JQ1 and iBET-151 treatment (Figure 2F).

To further investigate the role of FOSL1 in establishment of the MIR21-SE, we analyzed the promoter and enhancer region of MIR21 based on our FOSL1 ChIP-seq results of human SCC cells. Surprisingly, we found that FOSL1 was also significantly enriched in MIR21-SE region and MED1 occupancies on SEs in MIR21 were inhibited in cells treated with FOSL1 siRNAs (Figure 2A). ChIP-PCR results confirmed that the enrichments of MED1, BRD4 and FOSL1 on MIR21-SE region were significantly eliminated in cells treated with FOSL1 siRNA (Figures 2G-I). To further validate that FOSL1 can interact with the MIR21-SE, we cloned a fragment of MIR21-SE regions as well as a similar length negative control region into the pLG4.23 luciferase reporter. As shown in Figure 2J, knockdown of FOSL1 significantly suppressed the MIR21-SE luciferase activity. Consistently, the MIR21-SE fragment was also capable of elevating the luciferase reporter activity as compared to the negative control (Figure 2K). As expected, the expression of miR-21-5p was significantly inhibited in cells transfected with FOSL1 siRNA (Figures 2L, M). These findings support a notion that FOSL1 promotes miR-21-5p expression by interacting with MIR21-SE.

\section{Restoration of miR-21 Attenuates FOSL1 Depletion-Mediated Inhibition of Cell Proliferation and Invasion in HNSCC Cells}

To clarify the functional role of miR-21-5p in FOSL1-mediation cell proliferation and invasion, simultaneous knockdown of FOSL1 and restoration of miR-21-5p were performed in HNSCC cells. As shown in Figures 3A-F, FOSL1 depletionmediated inhibition of cell invasion and migration were attenuated by overexpressing miR-21-5p. Similar results were also observed in cell proliferation assay (Figures 3G, H). These findings indicate that miR-21-5p is involved in FOSL1-mediated invasion and proliferation in HNSCC in vitro.

\section{The Expression of miR-21-5p Is Positively Correlated With FOSL1 and Indicates Poor Prognosis in HNSCC}

Next, we analyzed the correlation of FOSL1 and miR-21-5p and then assessed their prognostic value in HNSCC. As shown in Figures 4A, B, FOSL1 expression was positively correlated with miR-21-5p expression in HNSCC, supporting the notion that
FOSL1 promotes expression of miR-21-5p at the transcriptional level. As expected, the expression of FOSL1 was upregulated in HNSCC as compared to the normal tissue (Figure 4C). The increased expression of FOSL1 was also observed in T3,4 stage HNSCC when comparing to T1,2 stage HNSCC (Figure 4D). Similar results were also observed in HNSCC patients with lymph node metastasis as compared to those without lymph node metastasis (Figure 4E). The survival analysis revealed that high expression of miR-21-5p and FOSL1 indicated a poor prognosis in HNSCC (Figures 4F, G). Moreover, HNSCC patients with high expression of miR-21-5p and FOSL1 showed the worst overall survival as compared to the other groups (Figure $\mathbf{4 H}$ ).

\section{DISCUSSION}

MiR-21-5p has been extensively studied in variety of cancers and can function as an oncomiR to promote malignant progression of cancer, including HNSCC (25). As a result, miR-21-5p has been proposed as a promising diagnostic and prognostic biomarker, as well as an attractive therapeutic target for cancer treatment (25). However, the regulation of miR-21 is not well understood in HNSCC. Increasing evidences imply that miR-21 expression is maintained by transcriptional and posttranscriptional regulation $(26,27)$. Notably, it has been reported that AP-1, Ets/PU.1, C/EBP $\alpha$, NFI, SRF, p53, STAT3 and $\mathrm{AR}$ binding sites were observed in the promoter region of the MIR21 gene, indicating that miR-21 expression is controlled by a transcriptional activator or suppressor $(26,28,29)$. In agreement with these findings, our data shows that FOSL1, a member of the AP-1 family, occupied the promoter region of the MIR21 gene and regulated miR-21 expression. Moreover, FOSL1 is frequently dysregulated in HNSCC and has a critical role in the invasive growth, metastasis and stemness of $\operatorname{HNSCC}(30,31)$. These results indicate that dysregulation of FOSL1 might exert its function by upregulation of miR-21-5p expression.

Recently, several studies implicating SEs have an important role in the regulation of ncRNAs, including miRNAs, circRNA and lincRNAs (32-34). As a new type of gene regulatory center, SEs are is often found to be positively correlated with oncogenes in cancer $(35,36)$. Strikingly, we found that a SE was formed around the MIR21 gene, which is enriched with FOSL1, indicating that miR-21 was controlled by FOSL1-associated SE in HNSCC. SEs are considered to be a large cluster of regulatory elements, which have a high binding capacity with transcriptional coactivators (such as BRD4, Mediator, CDK7 or EP300) as compared to typical enhancer binding, and SEs have high potential to activate their target gene transcription to control cell identity (37-40). To investigate the functional role of SEs in the regulation of miR-21-5p, JQ1 and iBET-151, two well-known BET inhibitors, were used to disrupt the SE (41). As expected, the expression of miR-21-5p was significantly suppressed in HNSCC cells upon treatment with JQ1 and iBET-151. Importantly, ChIP-qPCR data showed that the enrichments of MED1, BRD4 and FOSL1 were decreased in SE 
A

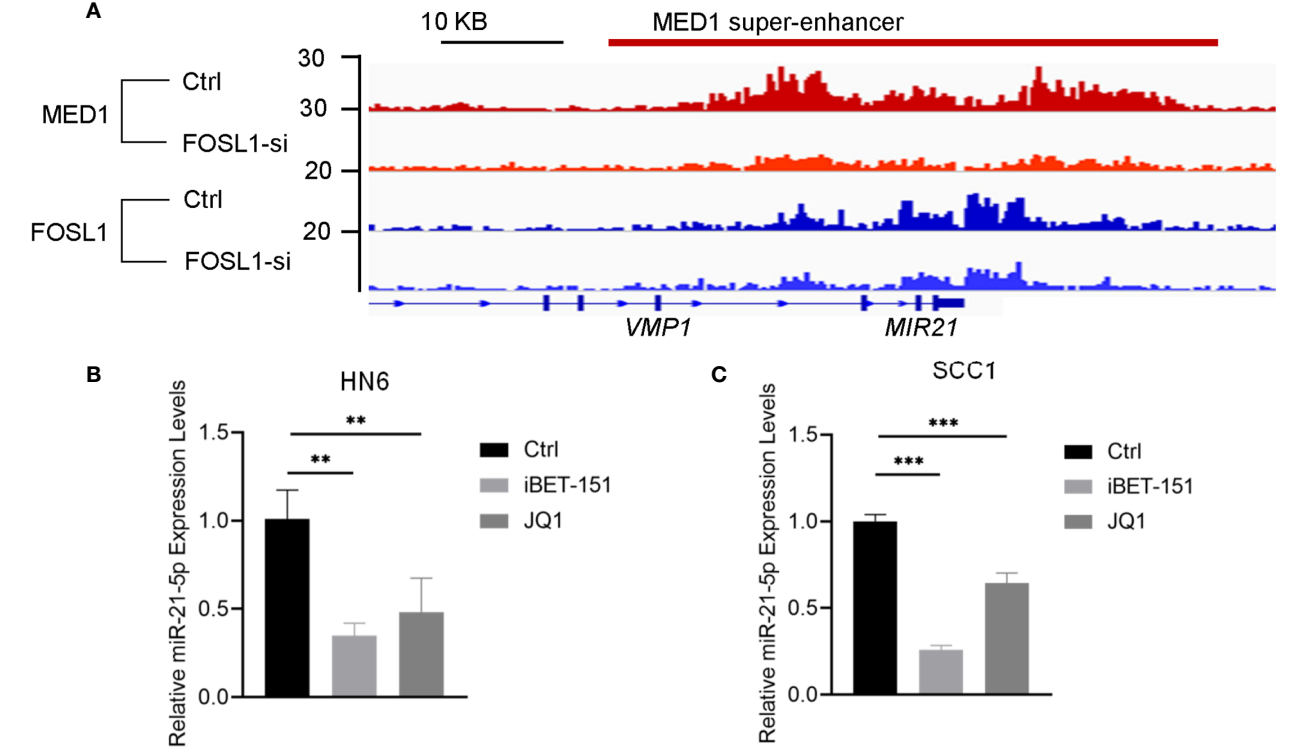

D

ChIP: MED1 E
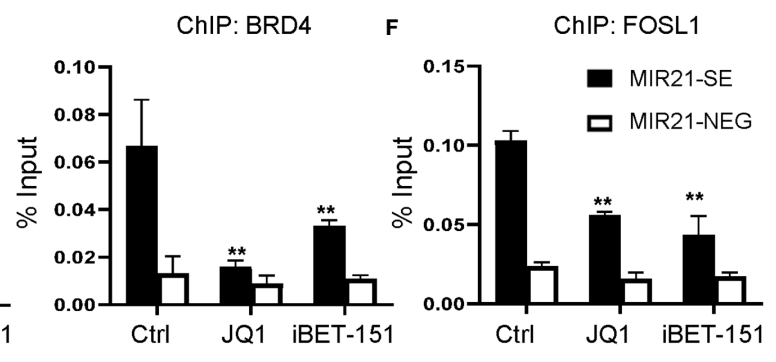

G
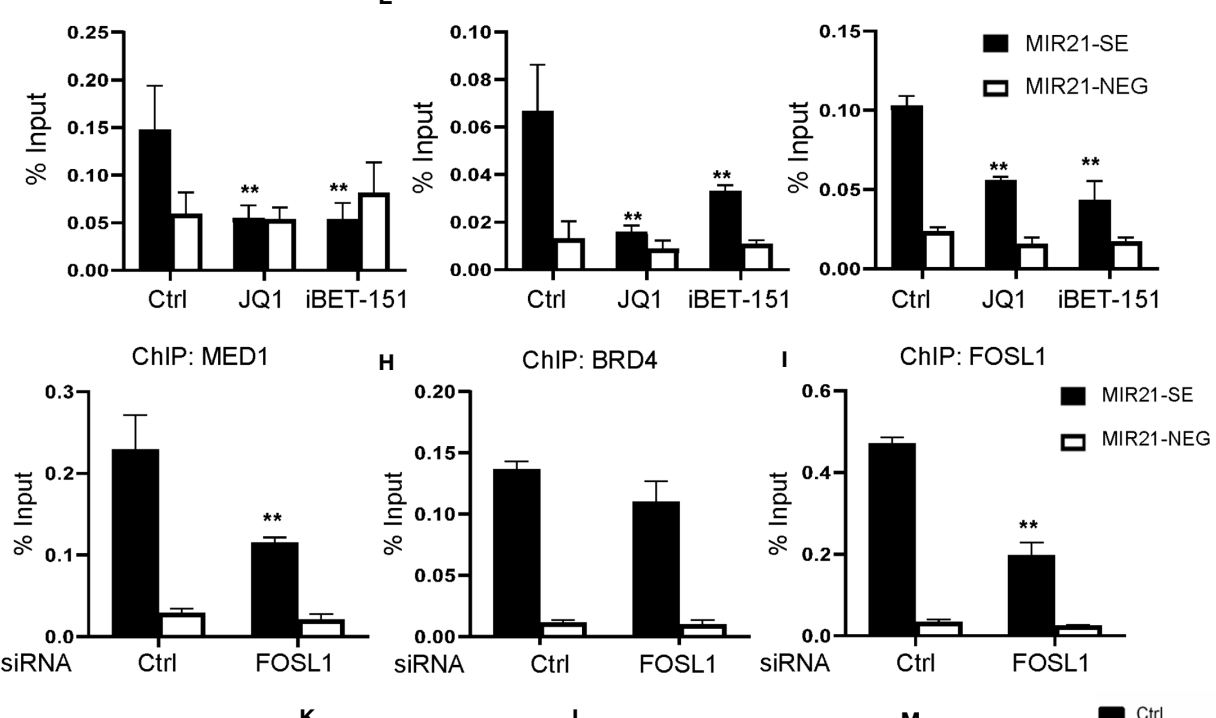

J

L
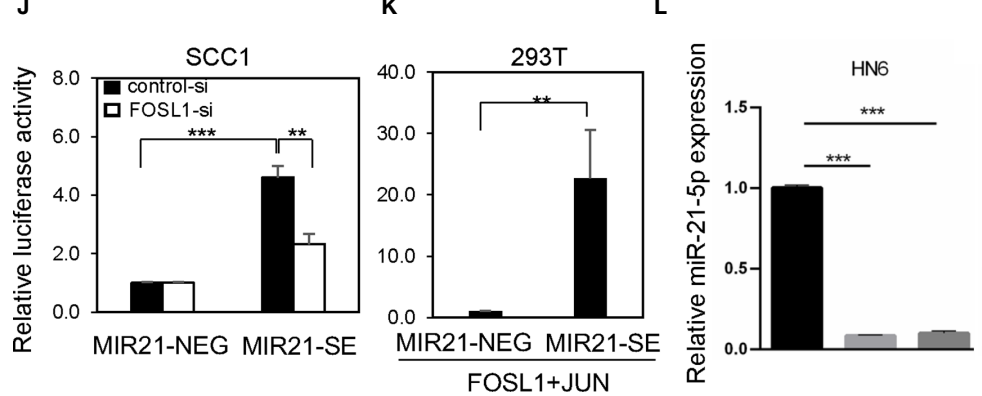

M

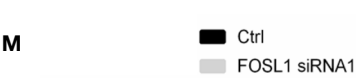

Ctrl

FOSL1+JUN

FIGURE 2 | Targeting FOSL1 suppresses miR-21-5p expression by interacting with the MIR21-SE. (A) ChIP-seq data revealed SE was formed around the MIR21 gene region and knockdown of FOSL1 suppressed the enrichment of MED1 and FOSL1 in the MIR21-SE. (B, C) Disruption of SE by JQ1 and iBET-151 inhibited the expression of miR-21-5p. ${ }^{\star *} \mathrm{P}<0.01,{ }^{* \star *} \mathrm{P}<0.001$ by one-way ANOVA. (D-F) The enrichments of MED1, BRD4 and FOSL1 in the MIR21-SE were eliminated in HNSCC cells upon JQ1 and iBET-151 treatment. ${ }^{*} \mathrm{P}<0.01$ by one-way ANOVA. (G-I) The enrichments of MED1 and FOSL1 in the MIR21-SE were eliminated in HNSCC cells treated with FOSL1 siRNA. ${ }^{*} P$ $<0.01$ by one-way ANOVA. (J) Knockdown of FOSL1 significantly suppressed the MIR21-SE luciferase activity. ${ }^{* * *} \mathrm{P}<0.001,{ }^{* *} \mathrm{P}<0.01$ by one-way ANOVA. (K) MIR21-SE fragment was capable of elevating the luciferase reporter activity as compared to the negative control. ${ }^{* *} \mathrm{P}<0.01$ by Student's t-test (L, M) Knockdown of FOSL1 inhibited the expression of miR-21-5p. ${ }^{* *} \mathrm{P}<0.01,{ }^{* \star *} \mathrm{P}<0.001$ by one-way ANOVA. 
A

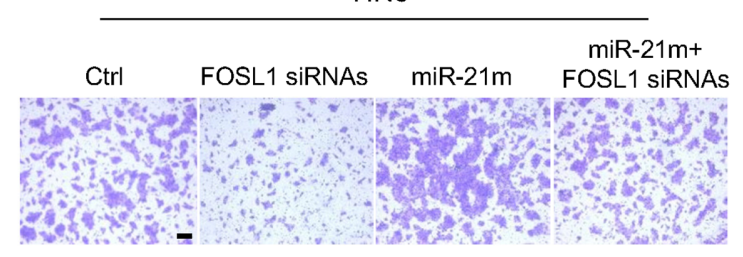

c

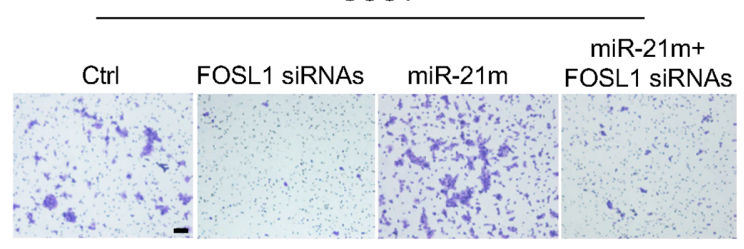

B

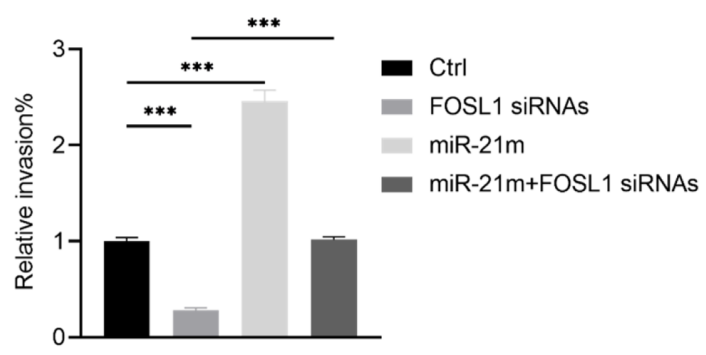

D

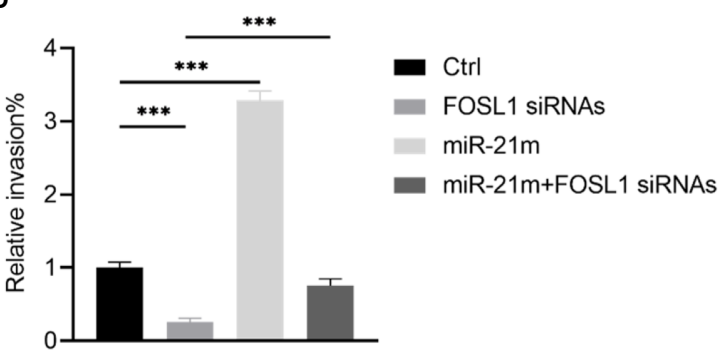

E

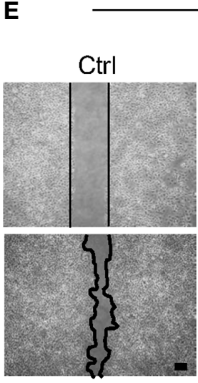

HN6

F

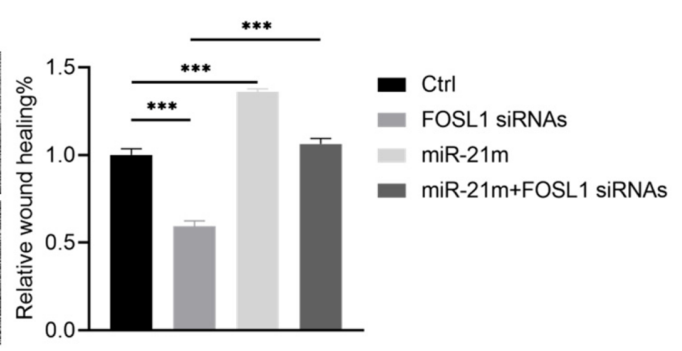

G

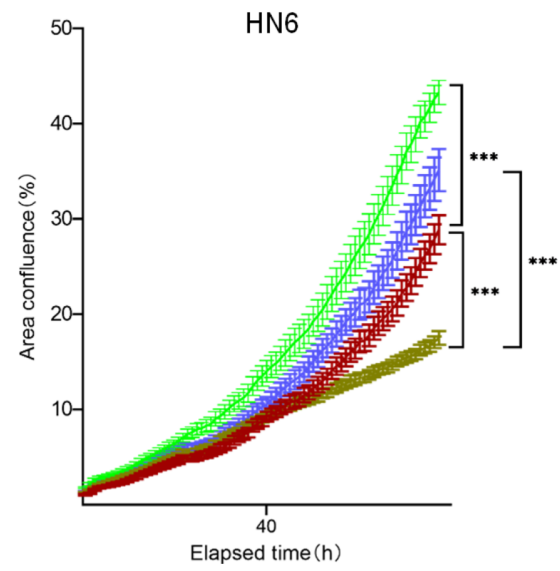

H

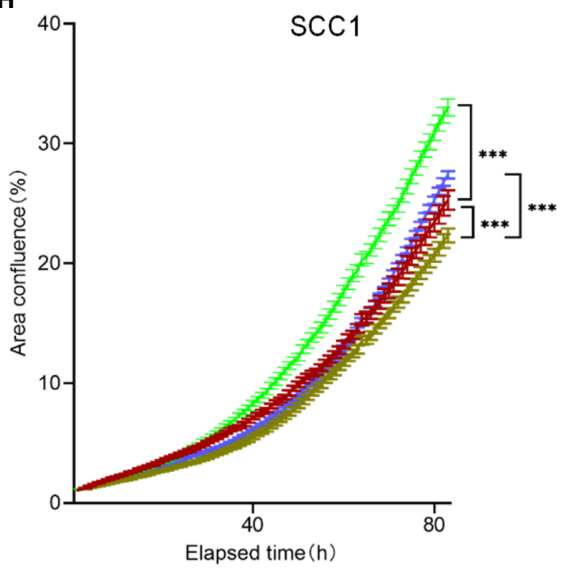

- Ctrl

- FOSL1 siRnAs

- miR-21m

- miR-21m+FOSL1 siRNAs

FIGURE 3 | miR-21-5p was required for FOSL1-medaited malignant progression in HNSCC. (A-D) FOSL1 depletion-mediated inhibition of cell invasion was attenuated by overexpressing miR-21-5p in SCC1 and HN6 cells. ${ }^{* \star} \mathrm{P}<0.001$ by one-way ANOVA. Scale bar, 200um (E, F) FOSL1 depletion-mediated inhibition of cell migration was rescued by overexpressing miR-21-5p in HN6 cells. ${ }^{\star \star \star}{ }^{\mathrm{P}}<0.001$ by one-way ANOVA. Scale bar, 200um (G, H) FOSL1 depletion-mediated inhibition of cell proliferation was impaired by overexpressing miR-21-5p in SCC1 and HN6 cells. ${ }^{\star \star \star} \mathrm{P}<0.001$ by two-way ANOVA.

region of MIR21. To further demonstrate the role of FOSL1 in SE, we knocked-down the endogenous expression of FOSL1 in HNSCC cells, the ChIP-seq and ChIP-qPCR results revealed that MED1 and BRD4 enrichment on the MIR21-SE were also decreased. In agreement with these findings, the expression of miR-21-5p was significantly decreased in HNSCC cells treated with FOSL1 siRNA, supporting the notion that miR-21-5p was controlled by FOSL1 driven SE in HNSCC. 
A

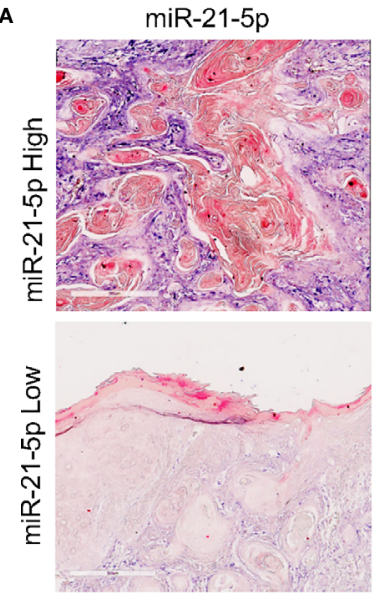

c

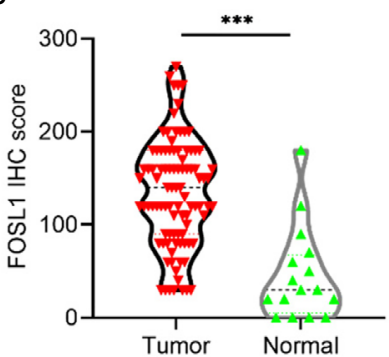

D

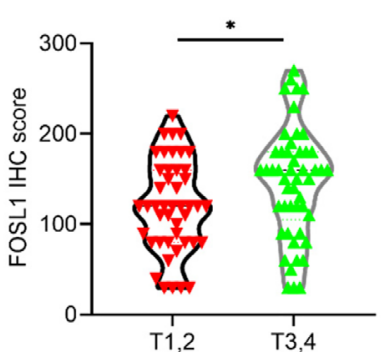

FOSL1
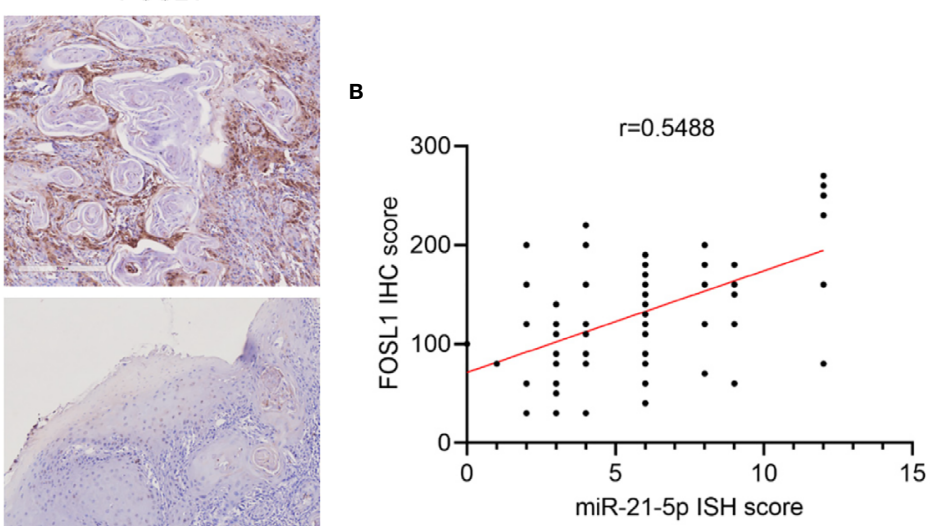

E

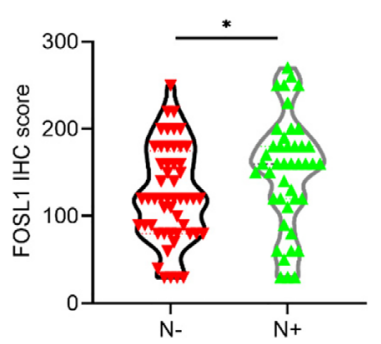

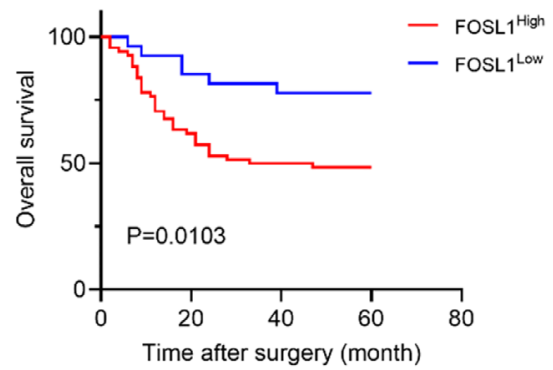

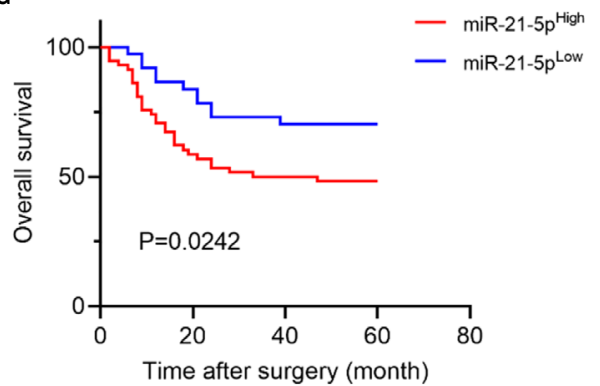

H

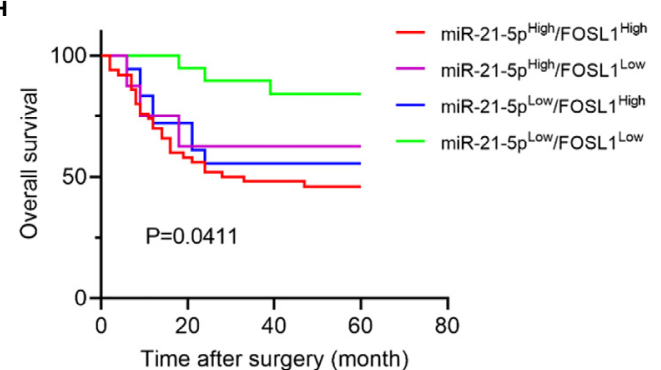

FIGURE 4 | miR-21-5p expression was positively correlated with FOSL1 in HNSCC. (A) The representative images of miR-21-5p ISH staining and FOSL1 IHC staining. Scale bar, 300 $\mu \mathrm{m}$ (B) FOSL1 expression was positively correlated with miR-21-5p expression. (C) The expression of FOSL1 was increased in HNSCC as comparted to the normal tissue. ${ }^{\star \star \star} \mathrm{P}<0.001$ by Student's t-test (D) The expression of FOSL1 was increased in T3,4 stage HNSCC patient as comparted to those with T1,2 stage. * $\mathrm{P}<0.05$ by Student's t-test (E) The expression of FOSL1 was increased in HNSCC patient with lymph node metastasis as comparted to those without lymph node metastasis. ${ }^{*} \mathrm{P}<0.05$ by Student's t-test. (F) HNSCC patient with high FOSL1 expression levels indicated a poor overall survival. (G) HNSCC patient with high miR-21-5p expression levels indicated a poor overall survival. (H) HNSCC patient with high FOSL1 and miR-21-5p expression levels indicated a poorest overall survival. 
Taken together, we identified a SE associated with the MIR21 gene driven by FOSL1 in HNSCC, which uncovers a novel mechanism underlying miR-21-5p regulation in cancer.

\section{DATA AVAILABILITY STATEMENT}

The datasets presented in this study can be found in online repositories. The names of the repository/repositories and accession number(s) can be found below: https://www.ncbi. nlm.nih.gov/, GSM4567094 and GSM4567097.

\section{ETHICS STATEMENT}

The studies involving human participants were reviewed and approved by Medical Ethics Committee of Hospital of Stomatology, Sun Yat-Sen University. The patients/participants provided their written informed consent to participate in this study.

\section{REFERENCES}

1. Pignon JP, le Maître A, Maillard E, Bourhis J. Meta-analysis of chemotherapy in head and neck cancer (MACH-NC): an update on 93 randomised trials and 17,346 patients. Radiother Oncol J Eur Soc Ther Radiol Oncol (2009) 92(1):414. doi: 10.1016/j.radonc.2009.04.014

2. Rêgo DF, Elias ST, Amato AA, Canto GL, Guerra EN. Anti-tumor effects of metformin on head and neck carcinoma cell lines: A systematic review. Oncol Lett (2017) 13(2):554-66. doi: 10.3892/ol.2016.5526

3. Amit M, Liu C, Gleber-Netto FO, Kini S, Tam S, Benov A, et al. Inclusion of extranodal extension in the lymph node classification of cutaneous squamous cell carcinoma of the head and neck. Cancer (2020). doi: 10.1002/cncr.33373

4. Wu ZH, Zhong Y, Zhou T, Xiao HJ. miRNA biomarkers for predicting overall survival outcomes for head and neck squamous cell carcinoma. Genomics (2020) 113(1 Pt 1):135-41. doi: 10.1016/j.ygeno.2020.12.002

5. Zhao X, Cui L. A robust six-miRNA prognostic signature for head and neck squamous cell carcinoma. J Cell Physiol (2020) 235(11):8799-811. doi: $10.1002 /$ jcp. 29723

6. Holt J, Walter V, Yin X, Marron D, Wilkerson MD, Choi HY, et al. Integrative Analysis of miRNAs Identifies Clinically Relevant Epithelial and Stromal Subtypes of Head and Neck Squamous Cell Carcinoma. Clin Cancer Res an Off J Am Assoc Cancer Res (2020) 27(3):831-42. doi: 10.1158/1078-0432.Ccr-20-0557

7. Koshizuka K, Hanazawa T, Arai T, Okato A, Kikkawa N, Seki N. Involvement of aberrantly expressed microRNAs in the pathogenesis of head and neck squamous cell carcinoma. Cancer Metastasis Rev (2017) 36(3):525-45. doi: 10.1007/s10555-017-9692-y

8. Diez-Fraile A, Ceulaer J, Derpoorter C, Spaas C, Backer T, Lamoral P, et al. Circulating Non-Coding RNAs in Head and Neck Cancer: Roles in Diagnosis, Prognosis, and Therapy Monitoring. Cells (2020) 10(1):48. doi: 10.3390/ cells10010048

9. Ren ZH, Wu K, Yang R, Liu ZQ, Cao W. Differential expression of matrix metalloproteinases and miRNAs in the metastasis of oral squamous cell carcinoma. BMC Oral Health (2020) 20(1):24. doi: 10.1186/s12903-020-1013-0

10. Zhang C, Cao W, Wang J, Liu J, Liu J, Wu H, et al. A prognostic long noncoding RNA-associated competing endogenous RNA network in head and neck squamous cell carcinoma. PeerJ (2020) 8:e9701. doi: 10.7717/peerj.9701

11. Zhuang Z, Yu P, Xie N, Wu Y, Liu H, Zhang M, et al. MicroRNA-204-5p is a tumor suppressor and potential therapeutic target in head and neck squamous cell carcinoma. Theranostics (2020) 10(3):1433-53. doi: 10.7150/thno.38507

12. Xie N, Wang C, Zhuang Z, Hou J, Liu X, Wu Y, et al. Decreased miR-320a promotes invasion and metastasis of tumor budding cells in tongue squamous cell carcinoma. Oncotarget (2016) 7(40):65744-57. doi: 10.18632/ oncotarget.11612

\section{AUTHOR CONTRIBUTIONS}

$\mathrm{CW}$ and JL conceived the study and designed experiments. YW, RH, $\mathrm{NX}, \mathrm{WW}, \mathrm{HC}, \mathrm{MZ}, \mathrm{GX}, \mathrm{ZM}, \mathrm{XX}, \mathrm{XL}$, and $\mathrm{ZH}$ performed the in vitro experiment, immunohistochemical staining and clinical analysis. CW, JL, YW, and RH analyzed data, wrote, and edited the manuscript. All authors have discussed the results and provided comments on the manuscript for improving the manuscript. All authors contributed to the article and approved the submitted version.

\section{FUNDING}

This work was supported by the National Natural Science Foundation of China (82073265, 81572661, 81772889), Guangdong Financial Fund for High-Caliber Hospital Construction (174-2018-XMZC0001-03-0125/D-14), and Natural Science Foundation of Guangdong Province (2017A030313515, 2017A030313558).

13. Zhuang Z, Xie N, Hu J, Yu P, Wang C, Hu X, et al. Interplay between $\Delta \mathrm{Np} 63$ and miR-138-5p regulates growth, metastasis and stemness of oral squamous cell carcinoma. Oncotarget (2017) 8(13):21954-73. doi: 10.18632/oncotarget.15752

14. Saleh AD, Cheng H, Martin SE, Si H, Ormanoglu P, Carlson S, et al. Integrated Genomic and Functional microRNA Analysis Identifies miR-30-5p as a Tumor Suppressor and Potential Therapeutic Nanomedicine in Head and Neck Cancer. Clin Cancer Res an Off J Am Assoc Cancer Res (2019) 25 (9):2860-73. doi: 10.1158/1078-0432.Ccr-18-0716

15. Manikandan M, Deva Magendhra Rao AK, Rajkumar KS, Rajaraman R, Munirajan AK. Altered levels of miR-21, miR-125b-2*, miR-138, miR-155, miR-184, and miR-205 in oral squamous cell carcinoma and association with clinicopathological characteristics. J Oral Pathol Med Off Publ Int Assoc Oral Pathol Am Acad Oral Pathol (2015) 44(10):792-800. doi: 10.1111/jop.12300

16. Chen Z, Jin Y, Yu D, Wang A, Mahjabeen I, Wang C, et al. Down-regulation of the microRNA-99 family members in head and neck squamous cell carcinoma. Oral Oncol (2012) 48(8):686-91. doi: 10.1016/j.oraloncology.2012.02.020

17. Garo Kyurkchiyan S, Miroslavov Popov T, Stancheva G, Rangachev J, Ivanov Mitev V, Petrova Popova D, et al. Novel insights into laryngeal squamous cell carcinoma from association study of aberrantly expressed miRNAs, IncRNAs and clinical features in Bulgarian patients. J BUON Off J Balkan Union Oncol (2020) 25(1):357-66.

18. Sun Z, Li S, Kaufmann AM, Albers AE. miR-21 increases the programmed cell death 4 gene-regulated cell proliferation in head and neck squamous carcinoma cell lines. Oncol Rep (2014) 32(5):2283-9. doi: 10.3892/ or.2014.3456

19. Kan X, Sun Y, Lu J, Li M, Wang Y, Li Q, et al. Co-inhibition of miRNA-21 and miRNA-221 induces apoptosis by enhancing the p53-mediated expression of pro-apoptotic miRNAs in laryngeal squamous cell carcinoma. Mol Med Rep (2016) 13(5):4315-20. doi: 10.3892/mmr.2016.5048

20. Zhang J, Hu H, Zhao Y, Zhao Y. CDRlas is overexpressed in laryngeal squamous cell carcinoma to promote the tumour's progression via miR-7 signals. Cell Proliferation (2018) 51(6):e12521. doi: 10.1111/cpr.12521

21. Kao YY, Chou CH, Yeh LY, Chen YF, Chang KW, Liu CJ, et al. MicroRNA miR-31 targets SIRT3 to disrupt mitochondrial activity and increase oxidative stress in oral carcinoma. Cancer Lett (2019) 456:40-8. doi: 10.1016/ j.canlet.2019.04.028

22. Qiang H, Zhan X, Wang W, Cheng Z, Ma S, Jiang C. A Study on the Correlations of the miR-31 Expression with the Pathogenesis and Prognosis of Head and Neck Squamous Cell Carcinoma. Cancer Biotherapy Radiopharmaceuticals (2019) 34(3):189-95. doi: 10.1089/cbr.2018.2621

23. Lu J, Tan T, Zhu L, Dong H, Xian R. Hypomethylation Causes MIR21 Overexpression in Tumors. Mol Ther Oncolytics (2020) 18:47-57. doi: 10.1016/ j.omto.2020.05.011 
24. Pfeffer SR, Yang CH, Pfeffer LM. The Role of miR-21 in Cancer. Drug Dev Res (2015) 76(6):270-7. doi: 10.1002/ddr.21257

25. Bautista-Sanchez D, Arriaga-Canon C, Pedroza-Torres A, De La RosaVelazquez IA, Gonzalez-Barrios R, Contreras-Espinosa L, et al. The Promising Role of miR-21 as a Cancer Biomarker and Its Importance in RNA-Based Therapeutics. Mol Ther Nucleic Acids (2020) 20:409-20. doi: 10.1016/j.omtn.2020.03.003

26. Kumarswamy R, Volkmann I, Thum T. Regulation and function of miRNA-21 in health and disease. RNA Biol (2011) 8(5):706-13. doi: 10.4161/rna.8.5.16154

27. Wang C, Peng R, Zeng M, Zhang Z, Liu S, Jiang D, et al. An autoregulatory feedback loop of miR-21/VMP1 is responsible for the abnormal expression of miR-21 in colorectal cancer cells. Cell Death Dis (2020) 11(12):1067. doi: 10.1038/s41419-020-03265-4

28. Fujita S, Ito T, Mizutani T, Minoguchi S, Yamamichi N, Sakurai K, et al. miR21 Gene expression triggered by AP-1 is sustained through a double-negative feedback mechanism. J Mol Biol (2008) 378(3):492-504. doi: 10.1016/ j.jmb.2008.03.015

29. Ribas J, Lupold SE. The transcriptional regulation of miR-21, its multiple transcripts, and their implication in prostate cancer. Cell Cycle (2010) 9 (5):923-9. doi: 10.4161/cc.9.5.10930

30. Chen D, Wu M, Li Y, Chang I, Yuan Q, Ekimyan-Salvo M, et al. Targeting BMI1(+) Cancer Stem Cells Overcomes Chemoresistance and Inhibits Metastases in Squamous Cell Carcinoma. Cell Stem Cell (2017) 20(5):62134 e6. doi: 10.1016/j.stem.2017.02.003

31. Ding X, Pan H, Li J, Zhong Q, Chen X, Dry SM, et al. Epigenetic activation of AP1 promotes squamous cell carcinoma metastasis. Sci Signaling (2013) 6 (273):ra28.1-13, S0-5. doi: 10.1126/scisignal.2003884

32. Suzuki HI, Young RA, Sharp PA. Super-Enhancer-Mediated RNA Processing Revealed by Integrative MicroRNA Network Analysis. Cell (2017) 168 (6):1000-14 e15. doi: 10.1016/j.cell.2017.02.015

33. Huang S, Li X, Zheng H, Si X, Li B, Wei G, et al. Loss of Super-EnhancerRegulated circRNA Nfix Induces Cardiac Regeneration After Myocardial Infarction in Adult Mice. Circulation (2019) 139(25):2857-76. doi: 10.1161/ CIRCULATIONAHA.118.038361

34. Xie JJ, Jiang YY, Jiang Y, Li CQ, Lim MC, An O, et al. Super-Enhancer-Driven Long Non-Coding RNA LINC01503, Regulated by TP63, Is Over-Expressed and Oncogenic in Squamous Cell Carcinoma. Gastroenterology (2018) 154 (8):2137-51 e1. doi: 10.1053/j.gastro.2018.02.018

35. Kim TM, Huang W, Park R, Park PJ, Johnson MD. A developmental taxonomy of glioblastoma defined and maintained by MicroRNAs. Cancer Res (2011) 71(9):3387-99. doi: 10.1158/0008-5472.Can-10-4117

36. Wang D, Gu J, Wang T, Ding Z. OncomiRDB: a database for the experimentally verified oncogenic and tumor-suppressive microRNAs. Bioinf (Oxford England) (2014) 30(15):2237-8. doi: 10.1093/bioinformatics/ btu155

37. Tsang FH, Law CT, Tang TC, Cheng CL, Chin DW, Tam WV, et al. Aberrant Super-Enhancer Landscape in Human Hepatocellular Carcinoma. Hepatol (Baltimore Md) (2019) 69(6):2502-17. doi: 10.1002/hep.30544

38. Di Micco R, Fontanals-Cirera B, Low V, Ntziachristos P, Yuen SK, Lovell CD, et al. Control of embryonic stem cell identity by BRD4-dependent transcriptional elongation of super-enhancer-associated pluripotency genes. Cell Rep (2014) 9(1):234-47. doi: 10.1016/j.celrep.2014.08.055

39. Sur I, Taipale J. The role of enhancers in cancer. Nat Rev Cancer (2016) 16 (8):483-93. doi: 10.1038/nrc.2016.62

40. Heinz S, Romanoski CE, Benner C, Glass CK. The selection and function of cell type-specific enhancers. Nat Rev Mol Cell Biol (2015) 16(3):144-54. doi: $10.1038 / \mathrm{nrm} 3949$

41. Di Costanzo A, Del Gaudio N, Migliaccio A, Altucci L. Epigenetic drugs against cancer: an evolving landscape. Arch Toxicol (2014) 88(9):1651-68. doi: 10.1007/s00204-014-1315-6

Conflict of Interest: The authors declare that the research was conducted in the absence of any commercial or financial relationships that could be construed as a potential conflict of interest.

Copyright (c) 2021 Wan, Hoyle, Xie, Wang, Cai, Zhang, Ma, Xiong, Xu, Huang, Liu, Li and Wang. This is an open-access article distributed under the terms of the Creative Commons Attribution License (CC BY). The use, distribution or reproduction in other forums is permitted, provided the original author(s) and the copyright owner(s) are credited and that the original publication in this journal is cited, in accordance with accepted academic practice. No use, distribution or reproduction is permitted which does not comply with these terms. 